Société d'histoire de la révolution de 1848 et des

révolutions du XIXe siècle

$22 \mid 2001$

Autour de Décembre 1851

\title{
Trois fraternelles de la région lilloise en 1849
}

\section{Lecompt}

URL : http://journals.openedition.org/rh19/259

DOI : $10.4000 /$ rh19.259

ISSN : $1777-5329$

Éditeur

La Société de 1848

Édition imprimée

Date de publication : 1 juin 2001

ISSN : 1265-1354

\section{Référence électronique}

C. Lecompt, «Trois fraternelles de la région lilloise en 1849 », Revue d'histoire du XIXe siècle [En ligne], 22 | 2001, mis en ligne le 27 juin 2005, consulté le 20 avril 2019. URL : http://journals.openedition.org/ rh19/259 ; DOI : 10.4000/rh19.259

Ce document a été généré automatiquement le 20 avril 2019

Tous droits réservés 


\title{
Trois fraternelles de la région lilloise en 1849
}

\author{
C. Lecompt
}

1 L'articulation adoptée ici n'est pas formelle; elle ne doit rien au calendrier. Tout au contraire elle répond à une réalité historique qui fait que, dès le départ de 1849 , dans la région lilloise au moins, l'histoire de la $\mathrm{II}^{\mathrm{e}}$ République s'infléchit progressivement dans une direction nouvelle, au terme de laquelle - et pour les Fraternelles ce sera décembre 1849 - on pourra mesurer tout le chemin parcouru depuis février 1948.

2 Le rapport que le préfet Durant adressait à Paris le 12 décembre, la grève de Roubaix terminée, était, on l'a vu, optimiste. Optimisme de commande ? En tout cas parfaitement inadéquat. Car, dès le début de 1849 , le patronat du Nord allait poursuivre l'effort de redressement ébauché par les filateurs roubaisiens à la fin de 48 .

3 Une première indication nous en est fournie par une lettre datée du 8 janvier, adressée au préfet par Debuchy au nom des fileurs et qui témoigne d'une vive amertume. Il y énumère toute une série de griefs: condamnation de deux fileurs "sans savoir pourquoi ", éviction de " 11 des nôtres", menace d'exclusion aux ouvriers qui refusent de quitter la Fraternelle, application d'un tarif établi par une commission où les fileurs n'étaient pas représentés et qui est tout à l'avantage des patrons, méconnaissance du maximum de 12 heures même par le maire "qui devrait donner l'exemple de la soumission aux lois". Et de conclure: "Si bien que nous pouvons déclarer tout haut que nous n'avons jamais été plus esclaves que sous la République" ${ }^{1}$.

4 Un tel bilan imposait à la Fraternelle un redoublement de vigilance et d'effort. Pourtant, en mars, Debuchy abandonne la direction de la société. L'explication est d'ailleurs facile ; elle permet aussi de mieux situer la personnalité et l'action du jeune Roubaisien.

5 Debuchy était, on l'a vu, très lié avec les hommes du Messager; il leur avait déjà apporté son concours lors des tentatives faites dans le Nord pour introduire la Solidarité républicaine ${ }^{2}$. Or, au début de 49 , les radicaux pensaient avant tout aux prochaines élections législatives, avec la conviction que, a les bien préparer, ils pourraient faire victorieusement appel de leur échec de 48. Encore fallait-il prospecter, atteindre tous les 
secteurs de la population; plus spécialement peut-être les milieux ouvriers dont l'éducation politique incomplète s'était fâcheusement révélée par le succès inattendu de Louis Napoléon aux élections présidentielles dans les grandes villes du Nord ${ }^{3}$. Dans ce sens, on procéda à une véritable division du travail. C'est ainsi qu'à Debuchy fut confiée la propagande destinée aux ouvriers et qu'il publia dans le Messager, au début du printemps, une série d'articles sous le titre de "Lettres d'un ouvrier à ses camarades du Nord" "

6 Ces articles sont assurément d'un bon radical. Mais, par delà les effusions républicaines, les lieux communs et la rhétorique qu'impliquait alors le style électoral, il est possible de discerner un accent personnel par quoi Debuchy révèle et son intelligence et sa connaissance des milieux industriels. En écrivant des journées de juin qu'elles ont été provoquées " par la faim et le désespoir des ouvriers ", il ébauche une explication qui sera élargie plus tard par le Messager ${ }^{5}$. Plus qu'aux industriels, c'est aux commissionnaires qu'il s'en prend. Il a la fierté de la classe à laquelle il appartient, qui vit du travail "seul titre de noblesse" qui doive subsister. Aux riches, aux blancs (car il les confond volontiers), il reproche leur esprit de classe qui leur fait considérer les pauvres comme d'une autre nature. Quand il parle de l'instruction gratuite et obligatoire et des multiples bénéfices qu'elle peut valoir à l'ouvrier, il donne l'impression de penser au profit personnel qu'il en a retiré. Son idéal de l'ouvrier est déjà celui du militant qui, non content d'avoir pris conscience de lui-même et de sa condition, s'emploie à éduquer ses camarades. Il conçoit la démocratie représentative sous une forme très directe: “Occupons-nous de rechercher les causes de nos misères, de trouver les moyens d'y remédier et lorsque nous aurons trouvé des abus, nous les ferons connaître à nos amis qui partent pour Paris. À nous de signaler les maux et les remèdes; à eux de les faire valoir à la tribune".

7 Dans ces élections de 1849, le Messager basait ses espérances de succès à la fois sur un large regroupement des forces républicaines dans le cadre de l'Union Démocratique opposée à la "Sainte Alliance du Nord" (formule du Messager); et sur le concours des masses prolétariennes du département. Dans ce sens, les radicaux avaient accentué le contenu social de leur programme et inscrit sur leur liste un plus grand nombre d'ouvriers (aucun n'appartient cependant aux fraternelles).

8 Le premier propos fut manqué puisque l'Écho du Nord s'obstina, sans grand succès il est vrai, à soutenir un tiers-parti " ni rouge ni blanc". Mais le deuxième objectif fut atteint car la liste d'union obtint d'excellents résultats dans les centres ouvriers, particulièrement à Lille, Roubaix, Tourcoing, Valenciennes. Les ouvriers contribuèrent ainsi et aux premiers rangs à l'incontestable succès remporté par la liste démocratique. Succès qu'il ne faut pas mesurer tellement aux six radicaux élus ${ }^{6}$; mais bien plutôt a ce fait que les dix-huit autres candidats du Messager se pressent en rangs serrés et exclusifs immédiatement en dessous du nombre de voix obtenues par le dernier élu radical luimême. Ce résultat était une raison de persévérer dans l'effort. Pour atteindre son but, le Messager comptait certainement sur les fraternelles. En tout cas, la collaboration ne cessa de se maintenir, en prenant d'ailleurs des formes variées et aussi très nuancées.

9 Des trois sociétés la moins active fut la Républicaine de Lille. Au vrai on ne relève de sa part aucune activité qui lui soit propre. On voit plutôt son action se diluer dans un effort plus large qui dépend avant tout des prud'hommes. Or, si la Société est représentée au sein de cet organisme, celui-ci subit essentiellement l'influence du Messager par le canal de son secrétaire, le radical Fémy, du brasseur Duthilleul (celui-là même qui, en 
novembre 1847, avait abrité dans sa propriété le fameux banquet de la Réforme) et de deux artisans lillois, Delmar et Schneider.

10 Une première initiative s'exprime dans la réponse faite par les prud'hommes au questionnaire ministériel touchant la création de caisses de secours et de retraite. Sans contester les bénéfices à attendre d'une telle institution, le Conseil affirme que l'essentiel est ailleurs; dans l'uniformisation des tarifs, dans la suppression de la retenue de la machine, dans la stricte application de la loi sur les heures de travail. Mais il y a plus. Car le document préconise également une politique industrielle dans laquelle il n'est peutêtre pas excessif de voir comme une ébauche du plein emploi et de la planification de notre temps. Il y est en effet recommandé de faire une statistique générale de la moyenne annuelle des produits industriels et du nombre d'ouvriers requis par cette production; et, si ce nombre dépasse les besoins du pays, de diminuer en conséquence la journée du travail ou bien de donner aux ouvriers en surnombre dans un secteur des encouragements et des secours qui leur permettent de trouver ailleurs une situation conforme à leurs devoirs de chef de famille et à leurs droits de citoyen ${ }^{7}$.

11 Un autre événement lillois intéresse directement la filature ; mais la Société Républicaine n'y joue aucun rôle apparent et ce sont de nouveau les prud'hommes qui prennent en charge la défense des ouvriers. Il s'agit d'un conflit entre un certain nombre de fileurs et les filateurs de coton à propos d'une initiative unilatérale de ces derniers, datant d'octobre, portant révision et unification des règlements d'atelier, ainsi que création d'une caisse de secours et de retraite alimentée par des retenues obligatoires sur les salaires. L'initiative patronale échoua par suite de l'opposition des prud'hommes soutenus sinon inspirés par le Messager. En retirant leur projet, les filateurs expliquèrent leur échec "par la politique qui vent se mêler d'une chose qui devrait rester tout industrielle" 8 .

12 La politique peut sans doute être invoquée ici. Mais aussi et surtout dans un autre sens, celui du durcissement croissant de l'opposition des partis en 1849. Et c'est ce même phénomène que nous allons retrouver à Tourcoing et à Roubaix.

13 A Tourcoing, la Fraternelle reste soumise a la double sollicitation qui peut s'incarner en Piscart et Leloire. De celui-ci, alors secrétaire des prud'hommes et, à ce titre, chef de file du prolétariat local, relève probablement une pétition des ouvriers de diverses industries, adressée au préfet, lui demandant de ne laisser accorder qu'exceptionnellement des dérogations a l'horaire légal de la journée de travail à cause du " péril imminent de voir tomber en désuétude une loi bienfaitrice ". Il est d'ailleurs peu de documents aussi directs et éloquents. Signalant la reprise des affaires, les signataires ajoutent : “ Nous voilà, nous, prolétaires, assurés du nécessaire [...] L'expérience nous crie : la continuation de notre bien-être est attachée a la limitation des heures de travail [...] Il faut savoir ce que nous souffrons dans les temps de crise pour comprendre combien nous sommes attachés aux mesures qui en préviennent le retour. Nous sommes, monsieur le Préfet, autant avides d'augmenter notre bénéfice que les patrons. (Rappelons qu'ils travaillent aux pièces). Mais nous craignons tant le retour de la misère, nous sommes si heureux de vivre une heure par jour de la vie de famille que nous vous supplions d'agir contre les infractions a la loi". Suivent les signatures, peu nombreuses, est-il ajouté, parce que beaucoup d'ouvriers ne savent pas écrire "qui n'ont jamais trouvé le temps de fréquenter l'école" 9.

14 Même a Tourcoing la favorable atmosphère de 1848 se détériorait. La démission du maire Masurel et du docteur Vanlaer, adjoint, en est probablement un signe ; du moins fut-elle déplorée par le Messager. Le patronat parait moins compréhensif ${ }^{10}$. Leloire semble céder 
davantage à l'influence de Piscart. Alors qu'en 48 un document l'avait désigné comme la "doublure de Debuchy", un rapport de police du 18 juin 49 l'appelle "l'acolyte de Piscart". Aussi l'activité de la Fraternelle, plus que corporative, tend-elle à devenir politique. Le même rapport de police déclare qu'elle fonctionne surtout à la veille des élections ${ }^{11}$. Elle est d'ailleurs l'objet d'une surveillance instante et une de ses réunions, interrompue par la police, donnera lieu à un rapport très courtelinesque où il est question de chansons républicaines inconnues chantées par "un homme à barbe de bouc" et de cris séditieux de: Vive la République démocratique et sociale! Piscart est toujours considéré comme le mauvais génie de la société. Étroitement surveillé, il est même renvoyé devant le Tribunal correctionnel et condamné à quinze jours de prison pour avoir, "étant en goguette", "battu horriblement" une jeune fille; enfin privé de sa charge de médecin du bureau de bienfaisance ${ }^{12}$. Un grand rapport du commissaire de Tourcoing, daté du 19 octobre, déclare que la Fraternelle a pu n'être pas dangereuse a l'origine, mais que, sous l'influence de Piscart, elle a fini par compter 1450 membres alors qu'il n'y avait à Tourcoing pas plus de 300 fileurs; qu'elle est devenue une société "bâtarde" de gens de toute sorte, comprenant jusqu'à des étrangers à la localité, toujours "des hommes de désordre" ${ }^{13}$. Ce rapport exerça une influence certaine sur la dissolution. Mais moins que la Fraternelle de Roubaix par une activité d'une exceptionnelle ampleur.

Le 10 septembre une grève éclate subitement à la filature de coton Motte-Bossut, au sujet des salaires. La Fraternelle ne semble pas en cause. On s'y arrêtera pourtant pour signaler deux faits par lesquels on peut mesurer toute la distance qui sépare l'automne de 49 de celui de 48. À cette grève le commissaire de police répond par des mesures autoritaires. Cinq instigateurs, convoqués et semoncés, sont, sur leur refus de reprendre le travail, incontinent incarcérés. Et le maire ajoute: “Cet acte de sévérité n'a pu vaincre leur résistance. Je viens de les visiter moi-même. Je n'ai rien pu obtenir”. (En fait les “ meneurs" allaient céder quelques jours plus tard).

16 Un deuxième fait n'est pas moins significatif. Dès le début de la grève, le maire Delattre avait demandé au préfet de déléguer un homme de confiance pour enquêter et terminer rapidement " cette malheureuse affaire ". Or le préfet lui répondit le 13, mais pour définir la nouvelle doctrine gouvernementale telle qu'elle était fixée par les instructions ministérielles du 2 février : l'Administration ne doit jamais s'immiscer dans la question des salaires, même si elle en était priée par les deux parties ; le taux des salaires obéit a la loi de l'offre et de la demande ; l'Autorité doit agir simplement par voie de conseil " pour éclairer les maîtres et les ouvriers sur leurs intérêts et leurs devoirs " tout en prévenant ou sanctionnant les fraudes et les violences. Le maire était donc prié de se conformer à ces instructions et, si besoin était, s'il était avéré qu'il y avait des faits délictueux, d'en aviser le procureur ${ }^{14}$.

17 Ce préfet n'est plus Durand Saint-Amand. Fin janvier, il a été remplacé par David. Le Messager pourra témoigner à ce dernier une courtoisie qui jure avec la sévérité qu'il avait fini par opposer à son prédécesseur. Il n'en reste pas moins qu'à un républicain de la veille a succédé un républicain du lendemain (ou du surlendemain). Car David, souspréfet de Guizot, avait été disgrâcié en février ; depuis il avait été nommé maire de Douai. Ce qui, ajouté à la nouvelle doctrine gouvernementale, crée aux grévistes des conditions moins favorables.

18 Ces grévistes sont, à partir du 15 septembre, les fileurs de la Fraternelle, car celle-ci entre alors en scène avec des méthodes originales et longuement mûries. La prévenance de 
quinzaine va servir de principe à l'interruption du travail. En d'autres termes, respectant l'usage des lieux, les grévistes ne vont quitter les ateliers qu'après avoir prévenu en même temps leurs employeurs et accompli la quinzaine réglementaire. Si bien que tout se passe comme s'il y avait grève sans coalition. Et c'est si vrai que l'Administration, désemparée, ne sait que faire. Le maire ira jusqu'à parler d" une apparence de légalité ". Et le préfet, en signalant que les filateurs désirent une action de l'Administration, écrira : “Ils n'ont rien précisé qui pût mettre sur la trace de la coalition qui existe évidemment, mais qui a, jusqu'ici, échappé à l'action de la justice ”.

19 La grève suit donc méthodiquement son cours. Successivement de nombreux ateliers sont abandonnés par les fileurs de la Fraternelle, les quinze jours de prévenance écoulés. Ceux du maire Delattre y échappèrent, où l'augmentation demandée avait été accordée. C'est que, dira le commissaire de police, M. Delattre - il est filateur et tisseur, occupant au total 700 ouvriers - vend directement ses produits sans passer par les commissionnaires ${ }^{15}$.

20 Il n'est pas facile d'évaluer l'importance numérique de cette grève. À la mi-octobre, quand le troisième échelon aura rejoint les précédents, il y aurait eu, selon une note de la préfecture, 421 grévistes (fileurs et rattacheurs compris), issus de nombreux ateliers dont quelques-uns étaient complètement arrêtés.

21 Cette méthode impliquait une longue durée, anormale pour l'époque, de la grève. Alors que, en ce même mois d'octobre, une grève des tullistes lillois échoue en quelques jours ${ }^{16}$, celle de Roubaix, commencée le 15 septembre, ne sera définitivement close que le 25 octobre. Et la question se pose de savoir comment les ouvriers, spécialement ceux du premier échelon, ont pu matériellement supporter une longue interruption de travail. La Fraternelle y veilla. Elle assura en effet aux grévistes une indemnité hebdomadaire de 12 francs. Et comme ses ressources normales ne lui permettaient pas de supporter une telle charge, elle éleva progressivement la cotisation de ses membres encore au travail à 4 francs par semaine, ce qui représente 20 fois la cotisation initiale et, approximativement, le septième du salaire du moment.

22 Tout dans cette grève, la discipline qui y présidait, les sacrifices consentis et plus encore l'habileté tactique, suppose une direction ferme et éclairée. Le maire reconnaissait que "des chefs bien intelligents" dirigeaient le mouvement. Il ne peut manifestement s'agir que de Debuchy. D’ailleurs, dans un rapport du 17 octobre, le procureur général affirmait que l'on savait désormais le rôle joué par Debuchy, "un des hommes qui obéissent aux inspirations du Messager" ${ }^{17}$. Celui-ci pourra faire valoir, lors de son deuxième procès, que depuis mars il n'avait pas repris ses fonctions de gérant; il n'en reste pas moins l'inspirateur de la Fraternelle et son action, ostensible dans l'affaire de Croix, suffirait à en donner une preuve incontestable.

23 Il importe de dire la cause de cette grève. En septembre les filateurs venaient d'admettre le relèvement du salaire horaire des rattacheurs. Or ce salaire étant payé par les fileurs, ceux-ci avaient à leur tour réclamé une augmentation de tarif. Les patrons s'y étaient refusés. Les documents de l'époque restent imprécis à cet égard. Mais on présumerait volontiers que les employeurs ont dû invoquer plusieurs augmentations antérieures qui avaient porté le salaire hebdomadaire du fileur jusqu'aux environs de 25 francs. Si bien que les avantages faits aux rattacheurs ont pu paraître aux uns la conséquence des relèvements antérieurs de tarif et aux autres la cause légitime de nouvelles revendications. La conjoncture économique de la fin de 49 peut d'ailleurs éclairer le problème. Après l'amélioration du début de l'année suivi d'un palier, dès la fin de l'été, les 
affaires avaient fortement repris. "Tout annonce, écrivait l'Écho le 29 septembre, une remarquable campagne d'hiver ". Il ajoutait que la façon de filature avait doublé et même au delà si bien que, les ateliers marchant à plein, la main-d'œuvre commençait à se faire rare ${ }^{18}$. Il n'est pas impossible que la Fraternelle pressente, entrevoie dès lors cette règle de la stratégie ouvrière selon laquelle les périodes d'euphorie économique sont les plus propices à une action revendicatrice.

24 Cette grève se déroulait dans l'ordre le plus parfait. Pourtant, dans les derniers jours de septembre, la région lilloise vécut des heures de lourde inquiétude et la bourgeoisie put se croire à la veille d'événements graves. C'est que, en effet, sur ce mouvement corporatif vint se greffer l'Affaire de Croix.

25 Le 20 septembre le maire de Croix recevait de Debuchy une lettre lui demandant, au nom de la Fraternelle, l'autorisation d'organiser un banquet dans sa commune le dimanche 30. Aux termes de cette lettre, il s'agissait de faire à cette occasion une convention entre les trois sociétés de Lille, Tourcoing et Roubaix afin que, en cas de manque de fonds de l'une d'entr'elles, la société en difficulté pût recourir aux caisses des deux autres; il était également question de modifier le règlement afin de donner des secours aux malades, "ce qui ne se fait pas présentement". L'engagement était pris par le secrétaire et les économes de ne pas troubler l'ordre public et de ne point parler des affaires du gouvernement. Et Debuchy ajoutait: “Je finis en vous donnant ma parole d'honnête citoyen que je ne répète ici que tout ce qui m'a été dit par les chefs et que, pour ma part, je suis prêt à répondre de l'ordre et de tout ce qui s'y fera " ${ }^{19}$.

26 L'initiative vient de Roubaix. Car, suivant une lettre de Debuchy au Messager, les fileurs de la Fraternelle de Roubaix s'assemblèrent le 15 août et " après délibération envoyèrent une lettre aux sociétés de Lille et de Tourcoing à l'effet de leur proposer un banquet fraternel et de faire alliance entre eux pour les besoins de leurs sociétés" ${ }^{20}$.

27 Avant de rien décider, le préfet voulut s'informer surtout auprès des maires de Tourcoing et de Roubaix. Cette correspondance et la presse locale permettent de poser et de tenter de résoudre les problèmes relatifs au banquet.

28 D'abord celui des mobiles. Les milieux conservateurs les ont recherché dans des intentions d'agitation politique et sociale. "C'est un effort du socialisme vaincu mais non découragé", écrit la Gazette de Flandre et d'Artois, organe légitimiste ${ }^{21}$. Et le maire de Roubaix d'estimer que, coïncidant "singulièrement" avec celles de plusieurs autres centres manufacturiers, cette grève impliquait un mot d'ordre général. En bref, il se serait agi d'une grande opération révolutionnaire projetée par les "démagogues". Interprétation assez gratuite que rien ne peut fonder sérieusement. Quant à établir un rapport direct entre la grève et le banquet comme inclinait à le faire le commissaire de police, la date du 15 août à laquelle le principe de la manifestation fut adopté impose d'expresses réserves. Au surplus Debuchy répond lui-même avec beaucoup de pertinence dans une lettre publiée par le Messager le $1^{\mathrm{er}}$ octobre :

29 “ On nous accuse d'organiser un banquet pour exciter les ouvriers à faire grève [...] Cette allégation est fausse; elle tombe d'elle-même quand je dirai qu'il serait imprudent, je dirai même maladroit, de faire un banquet pour exciter publiquement à faire grève alors que tout le monde sait que ces sortes de choses se font toujours en secret".

30 Il reste donc que les motifs invoqués sont plausibles. Toutefois les accepter sans plus serait se satisfaire de peu. Car, en définitive, il semble n'y avoir pas de commune mesure entre le caractère spectaculaire de la manifestation et les raisons techniques invoquées. 
Peut-être y eut-il l'ébauche d'un groupement organique des trois sociétés, d'un mouvement fédératif. L'exemple de l'Association Rémoise peut-il être invoqué ? Les seuls indices sont un article du Messager en date du 7 septembre qui mentionne l'Association, et une demande de renseignements du sous-préfet de Reims au préfet du Nord au lendemain de l'interdiction des Fraternelles ${ }^{22}$. Peut-être enfin pourrait-on penser que le banquet comportait des implications, des arrière-pensées politiques, le désir de maintenir dans la classe ouvrière un état de tension apte à la préparer à jouer son rôle dans le futur affrontement électoral des partis. (N'oublions pas que Debuchy est aussi un bon radical). On aurait donc ici une nouvelle expression de ce durcissement qui marque l'année 49.

31 Quoiqu'il en fût, même si le banquet n'était pas l'effet de ce durcissement, il devait, pour la région lilloise, en être une des causes principales.

32 L'annonce du banquet provoqua dans les milieux modérés une vague d'extrême inquiétude, significative de l'état de tension, de fébrilité qui s'accuse alors. Le maire de Tourcoing, " au nom des honnêtes gens, partisans de l'ordre ", adjure le préfet de refuser l'autorisation à une manifestation organisée par "les auteurs et fauteurs de troubles, les démagogues, les preneurs de doctrines anarchiques". Des bruits alarmants couraient que les rapports de police ne démentaient pas. Le commissaire de Roubaix annonçait que les fileurs iraient à Croix " en blouse et non rasés"; celui de Lille prévoyait un important contingent lillois, mais le voyait dans un autre appareil, formant " un peloton ayant des chapeaux gris". Aussi le préfet, après avoir interdit le banquet en vertu de la loi du 19 juin 1849, fit-il consigner un bataillon d'infanterie et un escadron de cavalerie afin d'être prêt à toute éventualité ${ }^{23}$.

33 Craintes vaines, car la journée du 30 fut très calme. Il y eut bien à Croix le rassemblement de 300 à 400 ouvriers venus surtout de Roubaix. Après s'être groupés, ils firent en chantant le tour de l'arbre de la liberté, entendirent quelques paroles, puis, se dispersant, allèrent terminer la journée dans les cabarets de la ville. L'ordre ne fut pas troublé un seul instant. Un rapport de police signale même que fort peu de manifestants se sont pris de boisson et qu'il a été facile de faire respecter l'heure réglementaire de fermeture des estaminets. Une telle sobriété, jointe à tant de discrétion, résulte manifestement de consignes très fermes.

34 En dépit de cette issue favorable, l'Affaire de Croix a marqué profondément la situation. Elle est au départ d'une crise, ouverte cette fois, qui atteignit bientôt son terme, c'est-àdire l'interdiction des Fraternelles.

35 Les ouvriers ont vu dans l'interdiction une véritable provocation. Pour Debuchy, en demandant au préfet d'empêcher la manifestation, "les gros bonnets de Roubaix" ont voulu exciter les fileurs à la révolte afin de détruire leurs sociétés et d'emprisonner leurs chefs. Mais " ceci est bien ingrat de leur part, car ils doivent se rappeler que ce sont ces chefs qui ont maintenu l'ordre dans la ville pendant les premiers mois de la révolution de févriers". Au surplus cette provocation "des blancs et des biens" est bien inutile parce que les ouvriers ont une arme plus forte que les baïonnettes, "le suffrage universel " ${ }^{24}$.

36 De leur côté, les modérés ont vu dans la rencontre de la grève et de la manifestation plus qu'une coïncidence : la double expression d'une volonté bien arrêtée de subvertir l'ordre établi. Si bien que, dès le début d'octobre, ils commencent une offensive qui, sous des formes variées et grâce à des concours divers, les conduit bientôt à un succès total.

37 Leur premier but était la fin de la grève. Le 5 octobre, le maire de Roubaix faisait afficher une proclamation qui, si elle en appelait à "la conscience des ouvriers", les menaçait 
aussi de la justice : "Elle a l'œil ouvert sur vous; elle saura vous atteindre et vous punir avec une juste sévérité". Dans le même temps, les employeurs recouraient à des moyens plus efficaces en engageant des ouvriers étrangers, belges pour la plupart. Selon le commissaire de Roubaix, du $1^{\mathrm{er}}$ au 24 octobre, les patrons embauchèrent 70 ouvriers. Ainsi, la lassitude faisant son œuvre, la grève se résorba progressivement et, le 24 , il ne restait plus que 30 à 35 ouvriers sans emploi ${ }^{25}$.

Cependant l'appareil judiciaire avait fini par entrer en mouvement. Un moment désemparé par l'habile tactique des fileurs, le Parquet avait trouvé dans les indemnités payées la preuve de la coalition et le moyen d'atteindre les responsables là où ils étaient, c'est-à-dire dans la Fraternelle ${ }^{26}$. D'où le 16 octobre, l'arrestation de Debuchy et de la plupart des économes de la société, tandis que les papiers étaient saisis et la caisse mise sous équestre ${ }^{27}$.

39 Les prévenus comparurent devant le Tribunal correctionnel de Lille au cours des audiences des 7 et 8 novembre. Le ministère public était occupé par un nouveau procureur, installé le 3 octobre, Pouillaude de Carnières ${ }^{28}$. Il mena l'attaque contre Debuchy avec une extrême vigueur, le désignant comme le constant animateur de la Fraternelle, allant jusqu'à l'appeler "le Mazaniello de Roubaix". $\mathrm{M}^{\mathrm{e}}$ Flament, cette fois encore défenseur de Debuchy, lut de larges extraits du règlement de la société ${ }^{29}$ et s'attacha à prouver que celle-ci n'avait que des buts très louables; il fit l'éloge du prévenu qui avait su s'attacher les fileurs au point que ceux-ci venaient de lui offrir de payer les frais de la défense; il déclara que Debuchy avait toujours usé de son influence au maintien de l'ordre, citant à ce propos une lettre à lui adressée par le commissaire de Roubaix : "Je compte, monsieur, sur un concours loyal de votre part pour le maintien de l'ordre et vous prie d'agréer mes salutations empressées". (Ce qui eut pour effet immédiat une intervention du procureur affirmant que l'auteur de cette lettre aurait à s'en expliquer ultérieurement). Enfin le défenseur, posant le problème politique, concluait: “Ce n'est point le fauteur de la coalition que l'on poursuit en Désiré Debuchy, c'est l'ancien secrétaire du club démocratique, c'est le républicain de la veille qui va dépensant sa jeunesse, publiant ses opinions, gagnant les cœurs à la cause démocratique $"{ }^{30}$.

40 Les deux grèves de 48 et 49 ne différaient guère, non plus que les responsabilités. Mais l'histoire avait progressé, l'atmosphère changé. Les neuf économes inculpés et Debuchy furent reconnus coupables du délit de coalition et, en application de l'article 415 du Code pénal, condamnés, " eu égard aux circonstances atténuantes qui existent au profit de tous ", celui-ci a dix mois de prison, ceux-là à des peines variant de huit jours à huit mois ${ }^{31}$. Cette double victoire (échec de la grève, condamnation de ses "moteurs") ne suffit pas aux partisans de l'ordre. Il leur parait en outre désirable et opportun de confirmer, d'élargir leur succès. Ce ne saurait être que par l'interdiction des fraternelles. Le maire Delattre a été formel: la société roubaisienne est pour lui " un foyer permanent de troubles et de désordre", "une association illicite et dangereuse qui tient tous les ouvriers dans une agitation permanente, produit un désordre continuel, exerce sur tous les industriels une pression fatale " ${ }^{32}$.

41 Mais le préfet David reste hésitant; effet sans doute de sa prudence naturelle et de ses scrupules de juriste. Car il ne suffit pas de tenir l'interdiction pour salutaire, encore fautil la fonder juridiquement. Or, à ce propos, la situation des fraternelles ne laissait pas d'être assez confuse. Ces sociétés pouvaient se réclamer de l'exception prévue en leur faveur par l'article $14 \mathrm{du}$ décret du 28 juillet 1848. Quant à leur appliquer la loi sur les 
clubs du 19 juin 1849, il fallait préalablement prouver qu'elles étaient devenues des réunions publiques de nature à compromettre la sécurité.

42 C'est à éclaircir cette situation que s'emploie le préfet durant le mois d'octobre. Il demande aux maires de Roubaix et de Tourcoing de lui fournir, en même temps que des exemplaires des statuts, des renseignements circonstanciés sur l'activité des fraternelles ; et la lettre qu'il adresse au procureur est une véritable consultation juridique ${ }^{33}$.

43 A voir la réaction des deux édiles, on a l'impression d'une véritable fuite devant les responsabilités. La réponse de Roubaix est très circonspecte lors même que le maire déclare la société dangereuse pour les grèves "et peut-être, le moment propice, pour organiser les émeutes ". Quant au maire de Tourcoing, bien qu'il eût assorti sa réponse du rapport de police déjà signalé, il concluait en disant que jusqu'ici on ne pouvait articuler aucun fait précis à l'encontre de la Fraternelle.

44 En cette occurrence, seul le procureur de Lille fit preuve de décision. En effet, l'épuration administrative que le journal réactionnaire la Liberté réclamait instamment depuis juillet, semble avoir commencé dans le Nord par le personnel judiciaire. Du moins, dès septembre, au procureur Ladureau nommé en mars 1848, avait succédé un ancien avocat général à la Cour de Douai, révoqué en février, Pouillaude de Carnières. Durant le procès de novembre 1849, il ne s'était pas contenté d'attaquer vigoureusement Debuchy; élargissant le problème, il avait été jusqu'à dire : “ Nous n'aurons ni paix ni trêve, nous ne prendrons pas un jour de repos que nous n'ayons brisé de pareilles associations qui sont autant d'obstacles à l'ordre et à la tranquillité publique " ${ }^{34}$. Dans sa réponse au préfet le procureur met fortement en valeur tous les arguments qui militent en faveur de l'interdiction; il n'hésite même pas à faire usage d'un rapport de police suivant lequel Debuchy et Leloire, s'étant rendu récemment en Belgique, auraient crié: "Vive la République démocratique et sociale! Vive la guillotine!" ${ }^{35}$. Bien plus, on a tout lieu de le tenir pour le promoteur d'une manœuvre qui vise à forcer la main au préfet hésitant. Le 26 novembre, le procureur général (plus exactement le premier avocat général faisant fonction) adressait au ministre de la Justice un long rapport qui, manifestement, s'alimente au Parquet lillois. Après avoir fait l'historique de la grève, insisté sur les dangers représentés par les fraternelles, signalé enfin les hésitations de David, il suggérait à la Chancellerie une intervention auprès du ministre de l'Intérieur. Le 30, Rouher donnait suite à cette suggestion. Il s'ensuivit une lettre de F. Barrot à David, en date du 6 décembre, "dans laquelle, usant de la formule impersonnelle " on m'informe", le ministre prescrivait la dissolution des fraternelles si, après enquête, les griefs qui leur étaient faits paraissaient fondés ${ }^{36}$.

45 Les événements rendirent cette intervention inutile. Entre temps, le ministère présidé par le général d'Hautpoul avait procédé à un important mouvement préfectoral. David, sacrifié, était remplacé par Vaïsse. Vaïsse est, lui aussi, une victime de février; il est pourtant autre chose qu'un républicain du lendemain. À le voir ministre de l'Intérieur dans le cabinet d'affaires du début de 1851, à considérer la brillante carrière qu'il fournira sous le Second Empire, spécialement à Lyon, on peut le tenir pour un homme de l'Élysée. Toujours est-il que, selon ses dires, venant à Lille, il mettait au premier rang de ses devoirs le rétablissement de la sécurité.

46 Le résultat ne tarda guère. Arrivé à Lille le 6 décembre, Vaïsse prononça la dissolution tant attendue le 12, après une information si rapide que, dans l'arrêté d'interdiction, il invoquera une grève "parmi les ouvriers fileurs de coton ". (Ce qui est probablement plus qu'un lapsus calami). L'arrêté atteignait les deux fraternelles; il était basé sur la 
condamnation du 17 novembre, sur les statuts qui "contiennent des dispositions de nature à provoquer le chômage et les grèves ". Si bien que, affirmant que les deux sociétés avaient cessé d'être des groupements industriels pour devenir des associations politiques, il ordonnait leur dissolution ${ }^{37}$.

47 Restait la Républicaine de Lille qu'on n'avait pas cru pouvoir impliquer dans la décision du 10 décembre. Pourtant la logique imposait de s'en débarrasser également. Le préfet en était bien convaincu. Encore fallait-il préparer une argumentation plausible. À cet effet un rapport fut demandé au commissaire de police lillois. Celui-ci récemment consulté par David avait répondu que la société n'entretenait aucune relation avec les fraternelles, qu'elle était essentiellement un organisme de secours mutuels, même si les membres les plus influents avaient été mêlés aux actes d'hostilité qui avaient opposé ouvriers et patrons depuis quelques temps ; donc que rien ne prouvait qu'elle menaçât l'ordre public. Or le même fonctionnaire envoie le 27 décembre un rapport d'une tout autre encre : la société qui compte 200 membres paraît fondée non pour secourir les malades mais pour soutenir les ouvriers en conflit avec leurs patrons; les cotisations ne servent qu'à entretenir l'oisiveté des chefs et la paresse ou le mauvais vouloir de ceux qui se font renvoyer des fabriques. Aussi demande-t-il la dissolution d'une société " qui renferme un élément de désordre" ${ }^{38}$. Quatre jours plus tard, Vaïsse publiait un arrêté dans lequel, invoquant le règlement de la Société Républicaine, son activité, ses liaisons avec les fraternelles dissoutes, se référant enfin au retrait d'approbation de Durand, il déclarait l'association interdite.

48 Ni la demande d'interpellation de Testelin à la Législative, ajournée de manière fort expéditive à trois mois (en fait sine die) ni les vives protestations du Messager accusant Vaïsse d'être "l'exécuteur des hautes œuvres des royalistes" n'y pouvaient rien ${ }^{39}$. Les arrêtés de dissolution furent, cette fois, strictement appliqués. Vaïsse s'employa même à interdire aux sociétés dissoutes de se reconstituer sous le couvert des anciennes mutuelles ${ }^{40}$.

49 Avec les fraternelles les fileurs ont perdu leur grand moyen de défense. Sans doute l'activité économique se relève assez pour que le chômage disparaisse et que les salaires se maintiennent à un niveau relativement favorable. Mais les obstacles opposés à certaines pratiques patronales révèlent alors toute leur fragilité; spécialement, an moment où la demande se fait très forte, la loi sur les heures de travail dont on a vu l'interprétation ouvrière. Un rapport du procureur général, daté du 6 février 1850, signale qu'“ il n'est sorte de moyens que l'on emploie pour éluder la loi...", on va même jusqu'à reprendre l'usage du travail de nuit " beaucoup plus nuisible à la santé et à la moralité " ${ }_{4}$ 0 bis.

50 Le département ne connaîtra en 1850 et 1851 aucune agitation notable. Mais, dans la région lilloise, le calme de la population ouvrière sera plus apparent que réel. Dès le 16 décembre 1849, dans son premier rapport périodique (qui n'est d'ailleurs qu'une copie d'un rapport de Pouillaude), le procureur général signalait bien que la condamnation des meneurs et l'interdiction des fraternelles valaient à l'arrondissement de Lille une situation satisfaisante; toutefois avec cette réserve que le Messager restait le foyer de toutes les mauvaises doctrines. Et il ajoutait: "Son influence [il parle de Bianchi] est encore très grande sur les ouvriers qui le considèrent comme leur père et qui, si le travail manquait, se jetteraient dans la lutte aveuglément et sans autre motif que sa volonté " ${ }^{41}$.

51 Des mesures complémentaires d'épuration parurent utiles. La première fut l'expulsion de Piscart, basée sur sa nationalité belge [12 octobre $1850^{42}$ ]. Après un court séjour en 
Angleterre auprès de Delescluze "son ancien ami et son conseiller" ${ }^{43}$, il vint s'établir, immédiatement au-delà de la frontière à Mont-à-Leux, dans un cabaret qui, selon la police, assurait la liaison entre les émigrés et les démocrates du Nord et de Paris. Mais la grande, la décisive épuration eut lieu au lendemain du coup d'État. La Commission mixte du Nord, bien qu'elle ait fait preuve de modération, prit un certain nombre de mesures à l'encontre des "rouges". Les principales visaient Bianchi, expulsé, et Debuchy, mis en résidence surveillée dans un autre département ${ }^{44}$. La police n'eut pas à exécuter ces sanctions. Les deux hommes en effet avaient déjà passé la frontière et gagné l'Angleterre pour y vivre dans le voisinage de Ledru-Rollin et de Delescluze. Bianchi rentrera à Lille après l'amnistie. Quant à Debuchy, la dernière information découverte jusqu'ici à son sujet ne dépasse pas le début de $1858{ }^{45}$.

52 Il importe maintenant de conclure. Et de conclure avec le souci, non seulement de dégager les traits originaux de cet effort ouvrier, mais aussi de replacer dans le cadre plus large de l'histoire nationale les événements qui, par les fraternelles, ont, en 1848 et 1849, affecté les trois grandes villes du Nord.

53 On signalera tout d'abord que le Nord a eu cette chance de trouver en Delescluze, Durand-Saint-Amand, David, Vaïsse enfin, quatre préfets qui par leur personnalité et leur tendance, caractérisent et rythment l'histoire de ces deux années cruciales. Peut-être même est-il possible d'ajouter que, du point de vue local, de ce rythme, la principale articulation se situerait dans l'hiver de 1848-1849, au moment où à la politique interventionniste succède le laisser-faire de la bourgeoisie libérale.

54 Mais l'essentiel de ces conclusions doit concerner les fraternelles, surtout celle de Roubaix.

55 On peut admettre qu'elles procèdent du compagnonnage, des mutuelles, bien plus encore des sociétés de résistance. Mais il est évident qu'elles vont beaucoup plus loin et qu'elles présentent, même avec ces dernières, des différences fondamentales. Car, au lieu d'une structure précaire, la plus représentative d'entr'elles a adopté des formes quasiorganiques; à une activité intermittente elle a substitué une action cohérente; ses méthodes n'ont plus été empiriques, mais ont témoigné d'un sens tactique déjà fort avisé ; et ce n'est plus à des résultats fragmentaires à quoi elle a visé, mais à imposer au patronat des accords généraux qui sont déjà des conventions collectives. La Fraternelle de Roubaix est avant la lettre un véritable syndicat ouvrier, organe de revendications corporatives réfléchies.

56 Cette ébauche d'un syndicalisme authentique a ses racines profondes dans les transformations de structure que subissait depuis quelques décades l'industrie textile; dans la naissance de la production capitaliste, et plus encore dans les conséquences, fâcheuses le plus souvent, qui en résultaient par l'interprétation bourgeoise du libéralisme économique pour les conditions d'existence du prolétariat. Mais il a fallu peut-être et surtout que, pour en prendre pleinement conscience, les fileurs aient subi les effets catastrophiques de la grande crise de 1846-1848, tout spécialement du chômage et de l'arbitrage patronal. On serait même enclin à se demander (avec toutes les précautions nécessaires) si cette crise, par ses multiples et durables conséquences, en révélant clairement les faiblesses du régime, n'a pas, mutatis mutandis, dans le XIX ${ }^{\mathrm{e}}$ siècle, une place un peu comparable à celle qu'occupe déjà dans le XX la crise de 1929. (Ne serait-ce que par la même crainte obsédante du chômage qui se maintint longuement). 
57 Quoiqu'il en soit, la Révolution de 1848 a dû à cet état de choses de dépasser largement ses causes initiales, de représenter ainsi dans l'histoire des révolutions une promotion qui fait d'elle la première des grandes crises des sociétés modernes. Les ouvriers du Nord, comme tous leurs camarades, ont eu l'espoir que février allait aboutir à des réformes capables de leur valoir un mieux-être ; ils y ont adhéré immédiatement.

58 Toutefois leur contribution a revêtu des formes variées. Dans un récent numéro de cette même revue. M. R. Gossez rappelait la thèse de M. Daniel Halevy suivant laquelle l'association ouvrière de production aurait détourné les travailleurs des sociétés de résistance, c'est-à-dire de leur moyen d'action le plus efficace ; mais pour la critiquer, en tendant à montrer que cette déviation n'est pas initiale, mais consécutive an drame de juin ${ }^{46}$. En s'appuyant sur l'expérience des trois Fraternelles de la région lilloise, ne conviendrait-il pas d'admettre, d'accentuer même cette critique ? En ce sens surtout que les deux formes, coopérative de production et société syndicale, ne s'excluent pas; qu'elles n'ont cessé de coexister. À constater l'écho faible et confus qu'eurent dans le Nord les événements de juin, nous serions même enclin à penser que si la formule coopérative l'emporte à partir du milieu de 1848 sur la formule syndicale, c'est moins. en conséquence de juin que du décret du 5 juillet 1848 , dont le principe est acquis dès le 9 juin. D'ailleurs ce décret n'a reçu dans le Nord que peu d'applications pratiques. Les demandes de subvention sont venues surtout de sociétés ou de patrons en difficulté; aussi ont-elles été rejetées et seule la Société Marre et Sourd a pu tenter sa chance ${ }^{47}$. En 1849, quelques coopératives de production se formeront par leurs propres moyens, telle la Fraternelle des cordonniers lillois ${ }^{48}$. Mais ni en 1848 ni en 1849 les fileurs ne seront un seul instant séduits par les perspectives de la coopération. N'est-ce point qu'il importe à cet égard de tenir compte des différences de structure industrielle? La coopération pouvait convenir à une structure artisanale; de là son importance dans la région parisienne. Elle était difficilement adaptable à la forme déjà capitaliste de la filature. En bref, parce qu'ils appartiennent a une industrie "progressiste", les fileurs du Nord se sont orientés vers la forme de défense ouvrière de l'avenir.

59 On ajoutera qu'une confusion doit être évitée. À comparer les mouvements ouvriers de mars et avril 1848 à ceux dont la Fraternelle de Roubaix a pris la responsabilité, il est difficile de ne pas remarquer une différence de nature. Les premiers sont contingents, dûs à des éléments disparates, spontanés, mais aussi désordonnés, ils risquent toujours de dégénérer en émeutes; ils traduisent l'ébranlement que février a provoqué dans les milieux ouvriers durement affectés par la crise; leur style est typiquement révolutionnaire. Les seconds sont réfléchis, organisés, disciplinés; ils n'affectent qu'une seule corporation ; jamais, en dépit des craintes des autorités, ils n'ont troublé l'ordre. Debuchy lui-même souligne la différence lorsqu'il objecte aux patrons la contribution des fraternelles, après février, au rétablissement de l'ordre dans la rue.

60 Si importante que soit en une telle occurrence l'infrastructure économique et sociale, un mouvement ouvrier d'une telle fermeté ne saurait se concevoir sans un principe d'action, une idéologie. À ce propos, on tient à signaler que, dès le départ de cette étude, on croyait trouver ce principe dans l'influence locale du socialisme. Et pourtant la conclusion qui s'est imposée en dernière analyse est en ce sens négative. On n'hésite pas à écrire que, dans la région lilloise, au début de 1848, il n'y avait pas de socialistes; du moins de socialistes authentiques. Sans doute des contemporains qualifient de ce terme certains de leurs compatriotes. Mais il n'est pas difficile de reconnaître en ceux-ci des démocrates, des radicaux qui aspirent sans doute à donner à leur démocratisme un contenu social, 
voire socialiste, mais qui n'y ont pas encore réussi. On peut trouver des preuves dans la position adoptée par le journal Le Peuple et par le club du même nom; avec, dans leur programme et la liste de leurs candidats d'avril 1848, beaucoup de confusion et d'imprécision, et comprendre ainsi la condescendance avec laquelle les traite le Messager 49. Une preuve est plus particulièrement significative. Des candidats à la présidence le plus spécifiquement socialiste était assurément Raspail. Or, dans tout le département, sur un nombre de votants qui atteint 213 350, dans le temps même où Ledru-Rollin, candidat du Messager et des fraternelles, obtenait 14441 suffrages, Raspail ne recueillait que le ridicule total de 38 voix.

61 Une telle carence du socialisme, alors que la région est une des plus industrielles et des plus prolétariennes de France, demande une explication. On la recherchait volontiers dans la fermeté du radicalisme lillois, dans ses fortes assises; plus directement encore dans le retentissement du banquet de la Réforme du 7 novembre 1847 par l'accent social que Ledru-Rollin avait mis dans son discours.

62 En définitive il ne saurait y avoir aucun doute : la seule source à laquelle ont puisé les chefs des fraternelles est le radicalisme de 1848, remarquablement représenté par les hommes du Messager. Mais cette conclusion revient bien moins à résoudre le problème qu'à le déplacer. Et ce serait négliger une question essentielle que de ne pas au moins tenter de fixer la position du journal radical par rapport au socialisme. Les années 1848 et 1849 peuvent servir de cadre à cette tentative.

63 En 1848 les hommes du Messager sont essentiellement des radicaux. Leurs valeurs, leurs modèles, ils les cherchent dans la grande tradition montagnarde de 1893. Assurément ils entendent donner à la démocratie un contenu social à la mesure de leur temps. En ce sens leur attitude est résolument interventionniste et leur hostilité complète à l'égard de "l'Économie politique" (les tenants du libéralisme économique). Mais, ce faisant, ils estiment être simplement dans la ligne de la démocratie jacobine ; et les réformes sociales nécessaires leur paraissent le surcroit naturel de la victoire républicaine, les seules capables d'ailleurs de donner à celle-ci sa pleine signification. Les valeurs dont ils se réclament restent la Liberté, l'Égalité, la Fraternité ; la Propriété également lors même qu'ils l'entendent contrôlée, élargie surtout pour y inclure le droit au travail ${ }^{50}$. D'autre part ils sont résolument réformistes et leur sympathie pour L. Blanc n'a d'égale que leur hostilité pour le communisme ${ }^{51}$. Enfin l'instauration d'une véritable république leur paraît ne pouvoir être obtenue que par le concours dévoué de tous les démocrates sincères, sans acception ni exception de classes, "car il n'y a qu'un peuple" 52.

64 On les sent en même temps très soucieux d'affirmer, de maintenir l'originalité du radicalisme. Le 14 mai 1848, dans le Messager, la Société républicaine radicale des Amis du Peuple, répondant à une proposition du club socialisme de Boulogne-sur-Mer d'établir une liaison permanente, disait : “ L'esprit de la Société républicaine [...] est démocratique radicale et non exclusivement socialiste [...] bien qu'elle accueille toujours avec plaisir l'expression des idées de cette école ". En septembre 1848, le Messager reproduit en bonne place le discours de Ledru-Rollin qu'animait un même souci et qui blâmait "la confusion systématique faite entre le socialisme et la République par les ennemis de la République ", et qui ajoutait : “On a dit : le droit au travail, c'est du socialisme. Je réponde : non, le droit au travail, c'est la République appliquée " ${ }^{33}$.

65 Mais l'année 1849 (c'est là une de ses caractéristiques essentielles) fut marquée pour le radicalisme par un glissement progressif d'une démocratie sociale à une démocratie socialiste. Dans cette évolution d'ailleurs classique par laquelle, le tiers-parti écrasé, la 
politique s'ordonne selon le couple "Mouvement " "Résistance ", le socialisme servira de principe de clivage. Si bien que désormais tout procédera du classement brutal: socialisme, antisocialisme. C'est en août 1849, à Limoges, que, dans ce dernier sens, Faucher lancera une formule destinée, sous des formes variées, à un brillant avenir : "Le socialisme, voilà l'ennemi à combattre ". Cependant que le mot socialisme devenait, à bon ou à mauvais escient, le terme générique dans lequel on enfermait toute la gauche.

En cette grave conjoncture, le Messager n'a en aucune hésitation : il est passé résolument à gauche pour constituer dans le Nord la force principale des "Rouges". Son évolution est presque terminée à la fin de 1849 où le journal oppose à l'Écho, s'obstinant dans sa position intermédiaire, l'exemple du National écrivant: "Républicains et socialistes sont aujourd'hui deux dénominations profondément synonymes "; et Marrast disant : “Je suis socialiste parce que je suis profondément républicain " ${ }^{54}$.

67 Toutefois cette évolution du Messager ne va pas sans quelque flottement. En avril 1849 le journal fait un large pas en avant en approuvant le programme collectiviste de la presse socialiste parisienne ${ }^{55}$. Plus souvent il met l'accent sur l'association de production ${ }^{56}$. Parfois il voit dans l'impôt progressif le principe essentiel par la vertu duquel, avec le temps, "la Révolution accomplira pacifiquement ses destinées" 57. On a l'impression que, dans cette vaste nébuleuse qu'est alors le socialisme, le Messager ne parvient pas à fixer sa position. Ses vues socialistes paraissent mal ajustées à son démocratisme initial; en quelque sorte surajoutées. Il lui manque sans doute d'avoir suffisamment repensé ses principes de base, d'avoir adopté une perspective appropriée aux besoins d'un monde nouveau, en bref de s'être élevé à une conception générale des problèmes du temps; on dirait volontiers sociologique. Aussi son socialisme très éclectique reste-t-il diffus, pins affectif que rationnel. Le Messager n'écrit-il pas: “Le socialisme n'est pas encore une science, c'est une aspiration, un sentiment " ${ }^{58}$. Et comme il s'adresse alors aux socialistes, on voit que, à cette date encore, il entend n'être pas confondu avec eux.

68 Il semble donc que le Messager a simplement atteint une position intermédiaire et quelque peu ambiguë; qu'il reste sollicité par l'alternative ou bien de revenir à sa conception initiale, purement radicale, ou bien de céder sous l'influence d'un ferment nouveau a une définitive mutation. Qu'on se souvienne que Guesde, en 1870, n'est guère plus qu'un radical et que seule la doctrine marxiste lui permettra d'aboutir à sa vocation socialiste.

69 A la fin de 1849, le Messager n'a pas encore atteint cette zone de clarté où le choix s'impose. On pourrait difficilement trouver plus révélateur de cette position qu'un article du 22 décembre. Répondant à ceux qui objectaient au socialisme la multiplicité et les contradictions des écoles le représentant, le journal écrit: "Le Suffrage universel, le Peuple souverain, régulièrement représenté par ses députés, choisira les bonnes propositions, repoussera les mauvaises, rectifiera les défectueuses et n'appliquera de mesures officielles que quand, dans sa sagesse, il aura élagué le mauvais du bon, l'ivraie du froment".

70 On voit donc que les radicaux du Nord restent encore attachés à leur mystique démocratique. Les problèmes du temps restent par eux posés en termes essentiellement politiques ; et, à les entendre inlassablement répéter que l'important est de bien voter, on pourrait même dire en termes électoraux.

71 Il reste à montrer dans cette perspective qu'elles furent à l'égard du Messager les réactions de chacune des fraternelles. Car le problème se pose. Voici en effet trois sociétés 
qui répondent à des facteurs économiques identiques; elles ont adopté des statuts presque semblables; et l'influence qu'elles ont subie est celle d'un même groupement politique. Or elles ont évolué différemment.

72 Des trois sociétés la plus importante est la Républicaine de Lille. Peut-être parce que, placé dans le voisinage immédiat du Messager, son bureau, formé de simples ouvriers, est resté sous la coupe de ce journal. Le milieu lillois a également joué son rôle; on y reviendra.

73 De la Fraternelle de Tourcoing, on dirait volontiers qu'elle s'incarne en Piscart. Et parce que celui-ci ne manque pas de personnalité ni d'autorité, il a donné à la société un incontestable dynamisme. Mais parce qu'il est aussi un bon radical, barrant Leloire et son réalisme ouvrier, il a accusé le danger de déviation politique qu'impliquait déjà l'influence du Messager. Aussi a-t-il fait prévaloir une conception plus politique que sociale des problèmes ouvriers. À la fin de 1849 , la fraternelle tourquennoise fait penser non pas à un organisme corporatif, mais à une société secrète ${ }^{59}$.

74 Si la société roubaisienne est de loin la plus intéressante, n'est-ce point justement pour avoir fait prévaloir les perspectives sociales? Pour le comprendre, il faut regarder vers Debuchy. Il aurait pu, lui aussi qui était bon radical, qui se réclamait de la grande tradition jacobine, devenir le Piscart de sa fraternelle. Pourtant, par lui, grâce à lui, celleci a en une action spécifiquement ouvrière.

75 On l'expliquera d'abord par les origines de Debuchy. Il n'est pas bourgeois, mais ouvrier, fils d'ouvrier. Jamais il n'a cherché à échapper à sa classe ; il est au contraire resté avec elle en contact étroit. Ainsi il a pu comprendre les travailleurs roubaisiens, leur inspirer confiance et même affection, devenir leur guide tout en obtenant d'eux la stricte discipline indispensable.

76 Mais à cette origine il faut joindre son intelligence, le réalisme de son intelligence. On ne prétend certes pas qu'il a eu la volonté, la conscience de tout ce qu'il a fait ou ébauché. On retrouve en lui la même ambiguïté que dans les hommes du Messager. À moins qu'il n'ait senti, sinon compris, que, au regard de la question ouvrière, il convenait de faire le départ entre les problèmes généraux et les problèmes corporatifs; ceux-là réclamant des solutions politiques, à longue échéance, ceux-ci, par leur particulière urgence, des moyens d'action immédiate. En tout état de cause, témoin et interprète compréhensif de son temps, on dirait volontiers de la nature et de la logique des choses, il a vu que l'industrie de la filature, par sa structure et par son évolution, imposait comme premier moyen d'action le groupement cohérent et organique des fileurs. Et il a orienté le mouvement ouvrier roubaisien vers la formule syndicale : par là, vers l'avenir.

77 Peut-être l'apport original du Roubaix de Debuchy pourrait-il se mieux comprendre encore à comparer, à opposer cette ville à Lille. Car elles sont fort différentes. Lille, avec ses 76000 habitants (sans compter ses faubourgs, Fives...) n'est pas seulement une cité plus peuplée. Elle a aussi une activité économique plus variée. Elle est enfin un foyer ancien de vie politique. Les industries modernes ont pu y prospérer largement, elle n'en conserve pas moins des activités artisanales très vivantes. Si bien que l'on y trouve juxtaposés deux types d'ouvriers différents : l'artisan et l'ouvrier de fabrique. Or si les radicaux comptent surtout sur les travailleurs de la grande industrie textile parce qu'ils sont le nombre ${ }^{60}$, ils font une place de choix aux artisans plus évolués. On en veut pour preuve le rôle qu'ils ont attribué à deux artisans, inscrits chaque fois sur la liste de leurs candidats aux élections législatives; en outre, membres en vue des prud'hommes: 
Léonard Delmar, ouvrier corroyeur, Schneider, ouvrier tailleur. Le premier avait d'ailleurs été, en 1833, membre de la Société des Droits de l'Homme ${ }^{61}$.

Roubaix, avec ses 34000 habitants en 1848, est au contraire une ville relativement récente. Sa population n'était encore, en 1830, date à laquelle elle a dépassé Tourcoing, que de 18000 . Elle s'est développée par et avec l'industrie de la laine grâce à une immigration qu'alimentaient les campagnards du plat pays et les frontaliers belges. L'ouvrier de fabrique y domine donc, plus fruste, moins ouvert aux problèmes politiques. Sans doute, par là, cédera-t-il parfois encore à l'influence du patronat ${ }^{62}$. Mais il peut aussi échapper plus facilement à la déviation politique lilloise et tourquennoise et participer avec plus de discipline à un mouvement corporatif.

79 En définitive, est-ce aller trop loin que de conclure que Debuchy annonce déjà, préfigure ces militants qui, sous le Second Empire, allaient tant contribuer à dégager le mouvement ouvrier de la confusion qui régnait avant 1848 , qui s'était encore maintenue durant la II ${ }^{\mathrm{e}}$ République, à définir les lignes directrices du syndicalisme moderne. On pense à Tolain (de la génération de Debuchy), à Varlin.

Assurément tout cet effort a abouti à un échec. Mais il n'est pas total. À la fin de 1849, alors que la police roubaisienne s'employait à mettre un terme aux dernières activités de la Fraternelle expirante, un des sociétaires, cédant à la contrainte, s'écriait: "Ils ne seront pas toujours les maîtres" ${ }^{63}$. Les rapports des procureurs généraux de Douai confirment la fidélité des ouvriers de la région lilloise à leurs chefs, même exilés, à leurs idées. On pourrait donc dire que les fraternelles ont comme sensibilisé les ouvriers du textile. À Roubaix peut-être plus qu'ailleurs. Et si la liaison reste à établir, peut-être n'estil pas trop téméraire de présumer d'ores et déjà que le choix fait en 1893 par Guesde de Roubaix comme circonscription n'est pas sans rapport avec l'atmosphère née en 1848 et 1849 et de penser que, par delà une certaine rhétorique électorale, dans la formule de remerciement du nouvel élu “ Ville sainte pour le prolétariat de tous les pays ", s'exprime l'implicite liaison du Roubaix de Guesde et du Roubaix de Debuchy.

\section{NOTES}

1. Voir $\mathrm{n}^{\circ} 188$.

2. Archives du Nord, M 139/21.

3. Messager, 13 décembre 1848.

4. Messager, 5, 11, 18, 25 mars ; 8 avril et 27 mai 1849.

5. Le Messager, fort embarrassé en juin, avait d'abord attribué le soulèvement aux intrigues royalistes et bonapartistes et approuvé Cavaignac. Mais le 30 octobre 1849, à propos de l'inauguration de la statue de Négrier à Lille, il écrivait : “En Juin, des deux côtés de la barricade, il y avait des républicains ".

6. Quatre d'entr'eux, il est vrai, avec le patronage complémentaire de l'Écho. Mais, à l'Assemblée, ils voteront régulièrement avec la Montagne.

7. Journal de Lille, 24 septembre 1849. 
8. Voir la presse lilloise des 11 et 25 octobre, ainsi que du début de décembre 1849 . Ce conflit, sa traduction dans la presse expriment parfaitement le degré d'opposition, d'incompréhension auquel on est alors arrivé. Cette atmosphère n'exclue d'ailleurs pas d'ultimes efforts pour résoudre amiablement certains problèmes, tel celui des heures de travail à Roubaix et à Tourcoing. Voir le Messager du 21 octobre; la Gazette de Flandre et d'Artois du 2 novembre 1849.

9. Messager, 22 août 1849.

10. Messager, 10 et 16 janvier 1849.

11. Archives du Nord, M 139/22.

12. Gazette de Flandre, 10 août ; Liberté, 26 septembre 1849. ROUSSEL et DEFONTAINE, ouv. cité p. 270.

13. L'article 2 des statuts ouvre la Société non seulement aux fileurs, mais "à tous ceux qui, par leur travail et leur emploi, sont en rapport direct avec la filature ".

14. Pour toute cette grève : Archives au Nord, M 620/16.

15. Voir également le Journal de Lille, 12, 19 mars ; 23 juin ; 27 juillet ; 15 novembre 1849.

16. Arch. nat., BB 18/1479.

17. Archives du Nord, M 620/16.

18. Messager, 15 novembre 1849.

19. Archives de Roubaix, série I, Affaires politiques $I^{\mathrm{e}}$ République.

20. Messager, $1^{\mathrm{er}}$ octobre 1849.

21. Gazette de Flandre, 26 septembre 1849.

22. Archives du Nord, M 595/3.

23. Archives du Nord, M 620/18.

24. Messager, $1^{\mathrm{er}}$ octobre 1849.

25. Archives du Nord, M 620/16.

26. Arch. nat., BB 18/1479.

27. Echo du Nord, 19 octobre 1849.

28. Journal de Lille. 10 septembre 1849.

29. Il signalait à cette occasion que la Fraternelle comptait 500 à 600 membres.

30. Presse lilloise des 15 et 17 novembre 1849 ; surtout le Messager.

31. Arch. nat., BB 30/360.

32. Archives du Nord, M 595/3.

33. Archives du Nord, M 595/3.

34. Messager, 17 novembre 1849.

35. Archives du Nord, M 595/3.

36. Pouillaude aura ultérieurement, sous des régimes fort différents, une brillante carrière ; directeur au ministère de la Justice de 1854 à 1861, commandeur de la Légion d'honneur, il sera nommé en 1862 conseiller à la Cour de cassation pour y devenir, de 1874 à 1882, président de chambre.

37. Arch. nat., BB 30/360.

38. Liberté, 17 décembre 1849.

39. Messager, 10 et 11 janvier 1850.

40. Archives du Nord, M 595/3.

4. ${ }^{0}$ bis Arch. nat., BB 30/377, 6 décembre 1848 et 6 février 1850. [II existe deux notes

“ 40 " dans le texte original]

41. Arch. nat., BB 30/377 (19 décembre 1849).

42. ROUSSEL et DEFONTAINE, ouv. cité p. 278.

43. Arch. nat., BB 30/377 (13 mars 1851). 
44. Arch. nat., BB 30/400.

45. Archives du Nord, M 140/27 et 141/76.

46. 1848 - Revue des révolutions contemporaires, $n^{\circ} 185, \mathrm{pp} .31$ et suivantes.

47. Office du Travail, Associations professionnelles ouvrières, tome 2, p. 591.

48. Le Travail affranchi, 14 février 1849.

49. Le journal Le Peuple est un quotidien qui parut à Lille du 19 mars au 26 avril 1848.

50. Messager, 20 avril, 19 juin, 22 septembre 1848.

51. Messager, 22 mai, 11 juin 1848.

52. Messager, 3 mai 1848.

53. Messager, 26 septembre 1848.

54. Messager, 29 septembre 1848.

55. Messager, 7 avril 1849.

56. Messager, 9 mars, 4 avril, 4 septembre, 10 septembre 1848.

57. Messager, 10 septembre 1849.

58. Messager, 10 novembre 1849.

59. Archives du Nord, M 595/3.

60. En y comprenant les filtiers qui sont d'ailleurs restés étrangers à cet effort d'organisation.

61. Journal de Lille, 29 mars 1848.

62. Messager, 30 octobre 1848.

63. Archives du Nord, M 595/3. 